\title{
ASSISTÊNCIA DE ENFERMAGEM EM UNIDADE PEDIÁTRICA: UMA PROPOSTA DE INÍCIO DE SISTEMATIZAÇÃO
}

\author{
Elizane Regina Santos* \\ Joziane Renata de Barros * \\ Marcia Maria Baraldi * \\ César Augusto Minto ** \\ Giselle Dupas ***
}

SANTOS, E.R. Assistência de enfermagem em unidade pediátrica: uma proposta de início de sistematização. Rev.Esc.Enf. USP, v.31, n.1, p. 36-50, abr. 1997.

O presente trabalho relata um processo inicial de sistematização da assistência de enfermagem desenvolvido em unidade pediátrica de um hospital de médio porte, no interior do estado de São Paulo, considerando os recursos humanos existentes e o tipo de abordagem adotada pela instituição na prestação da assistência à criança hospitalizada. Elaborou-se, com base na literatura e com a participação dos funcionários, um manual de rotinas e em seguida, realizou-se um treinamento desses funcionários, através de uma dinâmica grupal que procurou resgatar os conhecimentos que eles já possuím sobre os temas. O trabalho considerou a opinião dos funcionários sobre sua participação no processo de sistematização e treinamento.

UNITERMOS: Sistematização da assistência pediatrica; Dinâmica grupal participativa; Treinamento em Serviço.

\section{1 - INTRODUÇÃO}

"A criança é um ser biopsicossocial em crescimento e desenvolvimento e, como tal, deve ser atendida em toda a sua;individualidade, nas suas necessidades básicas de : nutrição, educação, socialização, afetividade. Durante o processo de desenvolvimento e crescimento, a criança está sujeita à apresentar afecções patologicas, que necessitam de uma hospitalização." (AUGUSTO; NODA, 1978)

\footnotetext{
* Alunas do curso de Enfermagem e Obstetrícia da Universidade Federal de São Carlos. UFSCar. Disciplina Estágio Profissional: Execução de Projetos.

** Bíblogo. Mestre em Didática. Professor Assistente do Departamento. de Metodologia de Ensino da UFSCar

*** Enfermeira. Mestre em Enfermagem. Professora Assistente do Departamento de Enfermagem da UFSCar.
} 
A cura das afecções da criança e o êxito do tratamento em um ambiente hospitalar não dependem exclusivamente do nível científico do pediatra, de um cuidadoso exame físico ou de uma perfeita prescrição médica; o trabalho da equipe de enfermagem é imprescindível. Ademais, uma conduta profissional integrada e co-responsável - médico/equipe de enfermagem - é de valor inestimável na tarefa de tratar e curar as crianças que chegam a necessitar de atendimento hospitalar, que por certo estaria potencializada se contasse com a participação ativa da família.

Segundo CAMPESTRINI (1991), a enfermagem vem desenvolvendo uma nova concepção de assistência integral ao indivíduo, onde novas responsabilidades devem passar a ser assumidas pela equipe de enfermagem, principalmente em algumas áreas especializadas, dando ênfase ao aperfeiçoamento de métodos, técnicas, normas e rotinas, com a finalidade de atingir o seu objetivo primordial: o bem estar da criança e sua reabilitação num tempo mais breve possível.

Durante a permanência da criança no hospital, ela deve ser atendida em todos os seus aspectos biopsicossociais. Considerando esses aspectos, SCHIMITZ et al (1989) detectaram três perspectivas diferentes que norteiam a assistência prestada à criança no processo saúde-doença. Segundo estas autoras, todo hospital adota uma abordagem de assistência à criança hospitalizada que, mesmo não estando explicitada em seus regimentos e manuais, pode ser facilmente identificada na rotina diária da unidade. Existem desta forma três tipos de abordagens: centrada na patologia da criança, centrada na criança e aquela centrada na criança e sua família.

A abordagem centrada na patologia caracteriza-se por ter como foco de assistência a criança com uma determinada patologia, sinal ou sintoma que necessitam de cuidados profissionais; neste caso, a equipe de enfermagem centra seus esforços para obter dados que se relacionam com os problemas de saude da criança, com o diagnóstico da patologia e com a instalação das medidas terapêuticas. As intervenções de enfermagem enquanto procedimentos técnicos, são o ponto alto da assistência. Na unidade, as crianças são geralmente agrupadas nas enfermarias conforme o diagnóstico médico. A área física em geral existe para atender as necessidades dos profissionais e o ambiente é pobre ou desprovido de caracterizações infantis.

A comunicação entre equipe, criança e família é formal, cabendo ao profissional de saude informar a família quando e o que julgar necessário. A interação da equipe de enfermagem com a familia resume-se a alguns momentos: admissão, comunicação de mudanças básicas no tratamento, agravamento do estado geral e alta hospitalar. A tomada de decisões é centrada no médico.

Já na abordagem centrada na criança o foco de assistência passa a ser a criança em sua unidade biopsicossocial. O objetivo dessa assistência é amenizar as repercussões psicológicas provenientes da hospitalização. Dá-se ênfase às necessidades de crescimento e desenvolvimento e às necessidades clínicas da criança, havendo também um incentivo maior à participação da criança e da familia nos cuidados, principalmente naqueles mais gerais como na higiene e alimentação. 
Quanto à área fisíca, destina-se locais para atender às necessidades de recreação e bem estar da criança, junto do acompanhante. A unidade passa também a ter caracterizações infantis.

A tomada de decisões torna-se mais democrática e a família, embora não participe dela, é mantida sempre informada e atualizada sobre a evolução do estado da criança, discutindo com os profissionais os resultados esperados.

$\mathrm{Na}$ abordagem centrada na criança e na familia, concebe-se a assistência como resultante da interação de fatores biopsíquicos, sócio-culturais e ecológicos. Visa a recuperar a saúde da criança promovendo as condições para evitar as intercorrências hospitalares e estender as ações à comunidade.

A área física é considerada como um local para estimulação da criança e para o convívio família-criança-equipe. O ambiente possui caracterizações infantis, condizentes com o objetivo de propiciar um bom estado de ânimo da criança, da familia e da equipe.

A família cumpre um papel central, participando ativamente do planejamento, execução e avaliação do atendimento. Os profissionais compartilham com ela, a identificação dos problemas, os recursos disponíveis e elaboram em conjunto um plano de assistência. Diferente das outras abordagens, esta é dinâmica, participativa e democrática. As decisões são tomadas por todos os membros e a responsabilidade é assumida de maneira igualitária pela equipe e família.

Entretanto, a maioria dos nossos hospitais, deficientes de recursos humanos e materiais, parecem adotar a abordagem centrada na patologia da criança, entendendo que ela dispende menor tempo para prestar assistência, economiza pessoal, e que o espaço físico da unidade mantém a organização, provocando menor estresse na equipe, pelo menor envolvimento com o paciente.

Independente do tipo de abordagem adotada, para obter-se uma assistência de enfermagem de qualidade, são indispensáveis a sistematização do serviço e a capacitação da equipe de enfermagem, sobretudo a dos atendentes de enfermagem que, segundo Dutra e Feldmann apud CURSINO (1992), constituem $60 \%$ da força de trabalho nas empresas prestadoras de serviços de saúde. A importância desses profissionais é vital porque eles são os principais responsáveis por manter as unidades funcionando vinte e quatro horas, durante todos os dias do ano.

Segundo KURCGANT et al (1991), as organizações hospitalares precisam de profissionais capacitados para alcançar as suas metas e objetivos. Necessitam não somente de processos seletivos adequados, mas também de trabalho contínuo com os funcionários, integrando-os na própria função e no contexto institucional. Para que isso ocorra, é imprescindível a educação continuada do funcionário no seu local de trabalho. Ela potencializa a atuação consciente desse profissional, além de oferecer condições para que ele interprete e utilize a realidade que $o$ cerca. Ela favorece a ação assistencial dos funcionários, levando-os a ter uma maior satisfação no trabalho, melhorando o seu desempenho profissional. 


\section{2 - JUSTIFICATIVA}

No decorrer do estágio da disciplina Enfermagem Pediátrica Clínica e Social, no $1^{\circ}$ semestre de 1994, na Unidade Pediátrica de um Hospital de médio porte, do interior de estado de São Paulo - local utilizado como campo de desenvolvimento das atividades práticas - percebemos a ausência de sistematização do serviço de enfermagem.

Entendemos que, a sistematização da assistência de enfermagem é um processo que deve abranger inúmeros aspectos. Dentre eles, a abordagem que permeia a assistência, os recursos disponíveis, a normatização de procedimentos, a formação continuada dos profissionais.

Diante da abordagem adotada pela Unidade - centrada na patologia da criança -; do interesse da instituição em investir no estabelecimento de rotinas manifestado pela enfermeira da Unidade; do quadro de funcionárias da Unidade - maioria atendentes de enfermagem, sem formação específica para o exercício da função; e do tempo disponível para a realização das atividades, nos propusemos a realizar um trabalho consubstanciado nos objetivos apresentados a seguir.

\section{3 - OBJETIVOS}

\subsection{Objetivos gerais:}

3.1.1 Deflagrar um processo de sistematização da assistência de enfermagem na Unidade Pediátrica em questão.

3.1.2 Elaborar rotinas acerca de procedimentos técnicos a serem utilizados na Pediatria, em conjunto com os profissionais que lá trabalham, e fornecê-las ao setor.

3.1.3 Treinar os funcionários segundo as necessidades mais urgentes da unidade.

3.1.4 Orientar os funcionários quanto à importância da atenção global à criança, através do enfoque dado durante o treinamento, visando com isso melhorar a qualidade da assistência à criança.

\subsection{Objetivos específicos:}

3.2.1 Estabelecer referenciais para execução de tarefas.

3.2.2 Iniciar a capacitação da equipe de enfermagem para a realização de procedimentos técnicos. 


\section{4 - METODOLOGIA}

\subsection{Caracterização do campo:}

4.1.1 Local: O campo utilizado no presente trabalho foi a Unidade Pediátrica de um hospital geral de médio porte, com capacidade de 350 leitos, localizado no interior do estado de São Paulo. A referida unidade atende crianças de 0 à 12 anos e conta com três alas, com duas enfermarias cada. Uma das alas é destinada à crianças portadoras de desidratação e/ou diarréia (20 leitos), outra (20 leitos) atende a crianças com problemas respiratórios. A terceira ala (20 leitos), destinase a pacientes pré e pós-cirúrgicos e crianças maiores. A unidade conta ainda com uma sala de medicação, refeitório, posto de enfermagem, isolamento (1 leito) e três quartos para atendimento de pacientes conveniados (9 leitos), além da ala particular que fica no andar superior (4 leitos), totalizando 74 leitos.

Apesar das condições precárias para acomodar acompanhantes, a mãe ou responsável fica junto com a criança em horários pré-determinados, das 9 às 12 e, das 15 ds 19 horas. Entretanto, parece-nos que a presença da mãe só se dá pelo dispositivo legal que lhe garante a presença, e não por uma questão de integração do familiar na assistência à criança.

4.1.2 Sujeitos: Trabalhamos com a equipe de enfermagem da unidade. As tarefas realizadas pelas funcionárias não são atribuídas de acordo com o nível de formação profissional. Tanto as auxiliares quanto as atendentes, realizam as mesmas tarefas e os mesmos procedimentos. Geralmente cada enfermaria fica sob a responsabilidade de uma funcionária e a medicação fica ao encargo de duas funcionárias, auxiliares de enfermagem ou então atendentes, mas com vários anos de experiência.

A equipe de enfermagem desta unidade é composta de 38 funcionárias fixas: uma (01) enfermeira que trabalha das 7 as $15 \mathrm{~h}$.; sete (07) auxiliares e vinte e nove (29) atendentes de enfermagem, que trabalham das 7 as $19 \mathrm{~h}$., em dias alternados. Além desses funcionários, há uma (01) auxiliar que trabalha das 19 às $24 \mathrm{~h}$. (Tabela 1).

\subsection{Procedimento de trabalho}

4.2.1 Estágio de Observacão: No decorrer do estágio da disciplina Enfermagem Pediátrica Clínica e Social, realizada no $1^{\circ}$ semestre de 1994 , tivemos a oportunidade de observar como as funcionárias realizavam os procedimentos, bem como as principais dificuldades na realização dos mesmos. Foi possível detectar também o tipo de abordagem que a unidade adota para nortear sua assistência à criança: a abordagem centrada na patologia.

4.2.2 Visitas a outras Unidades Pediátricas: Através da realização de visitas, tivemos a oportunidade de conhecer Unidades Pediátricas de hospitais-escola de outras cidades: - Hospital das Clínicas da Universidade de Campinas (UNICAMP); - Hospital das Clínicas da Universidade de São Paulo (USP) de Ribeirão Preto. 
Tabela 1 - Distribuição da equipe de enfermagem, segundo a carga horária de trabalho na Unidade Pediátrica

\begin{tabular}{|c|c|c|c|c|c|}
\hline & $\begin{array}{c}\text { Enfermeira } \\
(\mathbf{8 h})\end{array}$ & $\begin{array}{l}\text { Auxiliares } \\
\quad(12 \mathrm{~h})\end{array}$ & $\begin{array}{l}\text { Atendentes } \\
\text { (12h) }\end{array}$ & $\underset{(5 \mathbf{h})}{\text { Auxiliar }}$ & Total \\
\hline $\begin{array}{l}\text { Plantão } \\
\text { diurno diário }\end{array}$ & 1 & $\cdots$ & $\cdots \cdot$ & $\cdots$ & 1 \\
\hline $\begin{array}{l}\text { Plantão } \\
\text { diurno } \\
\text { dias pares }\end{array}$ & .... & 2 & 6 & ..... & 8 \\
\hline $\begin{array}{l}\text { Plantão } \\
\text { diurno } \\
\text { dias ímpares }\end{array}$ & $\cdots$ & 4 & 7 & $\ldots$ & 11 \\
\hline $\begin{array}{l}\text { Plantão } \\
\text { noturno } \\
\text { dias pares }\end{array}$ & $\cdots$ & 1 & 8 & $\ldots$ & 9 \\
\hline $\begin{array}{l}\text { Plantão } \\
\text { noturno } \\
\text { dias impares }\end{array}$ & $\ldots$ & $\ldots$ & 8 & $\ldots$ & 8 \\
\hline $\begin{array}{l}\text { Plantão } \\
\text { noturno diário }\end{array}$ & $\cdots$ & $\cdots$ & $\ldots$ & 1 & 1 \\
\hline Total & 1 & 7 & 29 & 1 & 38 \\
\hline
\end{tabular}

Nesses locais verificamos que a assistência de enfermagem é sistematizada, conhecemos suas rotinas, bem como a abordagem por eles utilizada no cuidado à criança.

4.2.3 Levantamento Bibliográfico: Realizamos um levantamento bibliográfico em livros, revistas e anais de literatura especializada, na Biblioteca Central da Universidade Federal de São Carlos e na Biblioteca Central da USP. Riberão Preto.

Após o estágio de observação, as visitas a outras Unidades Pediátricas e o levantamento bibliográfico, voltamos à Unidade Pediátrica em questão, para acompanhar os procedimentos de rotina - o que e como eram realizados, para detectar possiveis dificuldades.

4.2.4 Checagem e Listagem dos Procedimentos existentes na Unidade: Observamos e listamos os procedimentos que eram realizados diariamente e levantamos, através de observação direta, as principais dificuldades das funcionárias para realizar tais procedimentos. Alem disso, perguntamos às funcionárias quais as dificuldades que sentiam no desenvolvimento desses procedimentos. Através das informações levantadas, foi possivel nortear a elaboração do manual de rotinas e iniciar o treinamento.

4.2.5 Elaboracão do Manual de Rotinas: Com base nos itens anteriormente citados, elaboramos um manual prévio de rotinas, a ser discutido e reelaborado conforme as necessidades e possibilidades das funcionáris do local. Este manual foi composto por 21 rotinas que foram selecionadas por serem as mais realizadas 
na Unidade. Contém: considerações gerais sobre cada uma delas, material necessário para realização dos procedirnentos e sua descrição. Como a inserção do mesmo neste trabalho é inviável, devido à sua extensão (68 páginas), apontamos no Quadro I somente os Temas e Sub-temas que o compõem. A bibliografia consultada para a confecção do mesmo, consta no final deste trabalho.

Quadro 1 - Temas e Sub-Temas referentes ao Manual de Rotinas

\begin{tabular}{|c|c|}
\hline TEMAS & SUBTEMAS \\
\hline ADMISSÃO E IDENTIFICAÇÃO DA CRLANÇA & ANAMNESE, EXAME FÍSICO \\
\hline HIGIENE & $\begin{array}{l}\text { BANHOS DE: CHUVEIRO, IMERSÃO, LEITO } \\
\text { HIGIENE DAS MAOS E CORTE DE UNHAS } \\
\text { HIGIENE ORAL }\end{array}$ \\
\hline \multicolumn{2}{|l|}{ CONFORTO } \\
\hline ALIMENTAÇĀO & $\begin{array}{l}\text { ALIMENTAÇAO: DE CRIANÇAS PEQUENAS E POR } \\
\text { MAMADETRA }\end{array}$ \\
\hline SINAIS VITAIS E DADOS ANTROPOMÉTRICOS & $\begin{array}{l}\text { PESO, ESTATURA, PERIMETRO CEFÁLICO, } \\
\text { TEMPERATURA, FREQUENCIAS RESPIRATORIA E } \\
\text { CARDÍACA, PRESSÃO ARTERIAL }\end{array}$ \\
\hline \multicolumn{2}{|l|}{ APLICAÇŌES DE CALOR E FRIO } \\
\hline OBSERVAÇÖES DE ELIMINAÇÕES & $\begin{array}{l}\text { URINA (controle de eliminação através do saco coletor e da } \\
\text { pesagem diferencial das fraldas) } \\
\text { FEZES; VOMITOS; SECRECOOES EM GERAL }\end{array}$ \\
\hline RESTRIÇAO DE MOVIMENTOS & CONTENÇAO DE EXTREMIDADES \\
\hline ADMINISTRAÇÃO DE MEDICAMENTOS & $\begin{array}{l}\text { PRECAUÇÕES GERAIS; APLICAÇÃO TÓPICA; VIAS: ORAL, } \\
\text { NASAL, RETAL (supositório e enema); } \\
\text { PARENTERAL (id, sc, im, ev, soroterapia, dispositivo } \\
\text { intravenoso heparinizado) }\end{array}$ \\
\hline SONDAGENS & $\begin{array}{l}\text { NASOGÁSTRICA (alimentaçáo por gavagem, coleta de lavado } \\
\text { gástrico e lavagem gástrica); VESICAL; INTESTINAL }\end{array}$ \\
\hline OXIGENOTERAPIA & $\begin{array}{l}\text { CUIDADOS GERAIS; ADMINISTRAÇÁO POR: CATÉTER } \\
\text { NASAL, EXTRA.NASAL, TENDA (campânula, oxitenda, } \\
\text { croupette); INALAÇAO }\end{array}$ \\
\hline ASPIRAÇAO DE SECREÇÕES DE VIAS AEREAS & $\begin{array}{l}\text { SUPERIORES, TUBO ENDOTRAQUEAL E } \\
\text { TRAQUEOSTOMIA }\end{array}$ \\
\hline \multicolumn{2}{|l|}{ CURATIVO } \\
\hline $\begin{array}{l}\text { TROCA DE FRASCO DO SISTEMA DE DRENAGEM } \\
\text { TORÁCICA FECHADO }\end{array}$ & $\therefore$ \\
\hline CONTROLE HÍDRICO & INFUSÕES E DRENAGENS \\
\hline CUIDADOS COM EQUIPAMENTOS & INCUBADORAS, BERCO AQUECIDO E FOTOTERAPIA \\
\hline LIMPEZA & CONCORRENTE E TERMINAL \\
\hline \multicolumn{2}{|l|}{ OBSERVAÇÕES DE ENFERMAGEM } \\
\hline \multicolumn{2}{|l|}{ ANOTAÇOES DE ENFERMAGEM } \\
\hline \multicolumn{2}{|l|}{ PASSAGEM DE PLANTÃO } \\
\hline RECREAÇAO E BANHO DE SOL & \\
\hline
\end{tabular}


Dessas 21 rotinas, selecionamos 5 para o treinamento, mediante a observação de falta de habilidade técnica| das funcionárias e através da sugestão da enfermeira sobre as necessidades mais urgentes da unidade: Anotação de Enfermagem; Passagem de Plantão; Admissão da Criança; Verificação de Sinais Vitais (SSVV); Sondagem Nasogástrica (SNG).

\subsubsection{Treinamento das Funcionárias}

4.2.6.1 Planejamento do Treinamento das Funcionárias: Após a seleção das rotinas a serem trabalhadas, considerando o nível de escolaridade das funcionárias, que são na maioria atendentes de enfermagem e, portanto, possuindo apenas o primeiro grau, concluímos que o melhor método para realizar o treinamento seria através de aulas teórico-práticas com a utilização de dinâmica grupal participativa. Com o auxílio do Professor da Disciplina Prática de Ensino e Estágio Supervisionado em Enfermagem no 1e 2 Graus, planejamos algumas atividades nas quais buscamos resgatar as informações que as funcionárias já possuiam sobre o tema, iniciando com as experiências que elas já tinham, uma forma de construir ou reelaborar conjuntamente o conhecimento.

A proposta inicial foi treinar somente 4 funcionárias de cada plantão, para que as enfermarias não ficassem descobertas. Porém, a enfermeira sugeriu que fossem treinadas todas as funcionárias de cada plantão, assumindo a responsabilidade de estar presente na Unidade durante o treinamento.

Foram programadas 16 atividades no total. Todas as funcionárias seriam liberadas para participar das atividades tebrico-práticas a serem realizadas em dois períodos:

- Tarde - às $15 \mathrm{~h}$ (horário de visita), no Laboratório da UFSCar situado no próprio prédio do hospital;

- Noite - às 20:30 h (horário após a medicação) no refeitório da Pediatria.

O horário da tarde foi escolhido levando-se em conta que era o horário de visita da instituição e que poderíamos contar com os familiares, apos orientação dos mesmos, no cuidado com as crianças. O horário da noite foi determinado considerando a sugestão da própria equipe de enfermagem.

A equipe responsável por este projeto, juntamente com a enfermeira da Unidade Pediátrica, planejou a assistência que seria prestada às crianças durante o período de treinamento: ficaria ao encargo de duas das integrantes do projeto, com supervisão da enfermeira da Unidade. A primeira atividade sobre cada tema seria acompanhada pela docente da Disciplina Enfermagem Pediátrica Clínica e Social e, pelo docente da Disciplina Prática de Ensino que, assistindo a essas atividades dariam sugestões para as seguintes. Quando a atividade fosse realizada com as turmas seguintes, a docente de Enfermagem Pediátrica, assumiria a supervisão da Unidade para que a enfermeira também pudesse participar do treinamento.

Após o planejamento de conteúdos, dos horários, e da preparação das condições para a realização do treinamento, divulgamos tal trabalho através de 
cartazes colocados na Sala de Medicação e no Posto de Enfermagem da Unidade Pediátrica, expondo os temas, horários e locais de realização.

\section{5 - RESULTADOS}

5.1 Manual de Rotinas: O Manual de Rotinas foi elaborado pensando em sua aplicabilidade no campo, portanto, os procedimentos trabalhados fazem parte da rotina diária da Unidade. Todos os procedimentos abordados são específicos da Unidade em questão, levando em conta suas características, seus usuários, seus funcionários.

No manual não estão descritos procedimentos especializados e mais elaborados, já que a Unidade não atende crianças de alto risco, pois estas são atendidas na UTI infantil.

Embora os procedimentos de enfermagem fossem realizados diariamente, não havia contudo uma padronização e parte dos nossos objetivos foi elaborar um instrumento para viabilizar a realização de rotinas, oferecendo subsídios para que estas ocorressem de forma correta e sistemática.

5.2 Treinamento das funcionárias: Tal treinamento ocorreu segundo a disposição expressa na Tabela 2.

Tabela 2 - Distribuição do treinamento das funcionárias segundo data, tema abordado, horário e $\mathbf{n}$ de participantes.

\begin{tabular}{|c|c|c|c|}
\hline DATA & TEMA DA ATIVIDADE & HORÁRIO & $\begin{array}{c}\mathrm{N}^{\circ} \mathrm{DE} \\
\text { PARTICIPANTES }\end{array}$ \\
\hline $\begin{array}{c}21 / 11 / 1994 \\
e \\
28 / 11 / 1994\end{array}$ & $\begin{array}{l}\text { Observaçбes e } \\
\text { Anotaçzes de } \\
\text { Enfermagem }\end{array}$ & $\begin{array}{l}15: 00 \text { às } 15: 50 \mathrm{hs} \\
20: 30 \text { às } 21: 20 \mathrm{hs} \\
15: 00 \text { às } 15: 50 \mathrm{hs} \\
20: 30 \text { às } 21: 20 \mathrm{hs}\end{array}$ & $\begin{array}{l}11 \\
11 \\
07 \\
05\end{array}$ \\
\hline $\begin{array}{c}22 / 11 / 1994 \\
e \\
29 / 11 / 1994\end{array}$ & $\begin{array}{l}\text { Sinais Vitais, Admissão } \\
\text { e Identificaça da } \\
\text { Criança }\end{array}$ & $\begin{array}{l}15: 00 \text { às } 15: 50 \mathrm{hs} \\
20: 30 \text { às } 21: 20 \mathrm{hs} \\
15: 00 \text { as } 15: 50 \mathrm{hs} \\
20: 30 \text { ds } 21: 10 \mathrm{hs} \\
21: 15 \text { as } 21: 45 \mathrm{hs}\end{array}$ & $\begin{array}{l}09 \\
09 \\
06 \\
04 \\
05\end{array}$ \\
\hline $\begin{array}{l}23 / 11 / 1994 \\
25 / 11 / 1994 \\
30 / 11 / 1994\end{array}$ & $\begin{array}{l}\text { Passagem de Plantão e } \\
\text { Precauçซes Universais(1) }\end{array}$ & $\begin{array}{l}20: 30 \text { às } 21: 20 \mathrm{hs} \\
15: 00 \text { às } 15: 50 \mathrm{hs} \\
15: 00 \text { às } 15: 50 \mathrm{hs}\end{array}$ & $\begin{array}{l}10 \\
08 \\
07\end{array}$ \\
\hline $\begin{array}{c}24 / 11 / 1994 \\
e \\
01 / 12 / 1994\end{array}$ & $\begin{array}{l}\text { SNG, Lavagem Gástrica } \\
\text { e Lavado Gástrico }\end{array}$ & $\begin{array}{l}15: 00 \text { ds } 15: 50 \mathrm{hs} \\
20: 30 \text { ds } 21: 20 \mathrm{hs}\end{array}$ & total: 14 \\
\hline
\end{tabular}

(1) Este tema, apesar de não constar do Manual de Rotinas, emergiu durante as atividades e, por esse motivo, foi abordado com todos os grupos 


\section{6 - AVALIAÇÃo}

A proposta de treinamento de todas as funcionárias dos quatro plantões não ocorreu da forma planejada devido a intercorrências no decorrer do processo. Entretanto, a maioria delas esteve presente nas atividades previstas.

Durante o treinamento todo, após cada atividade, ocorreram reuniões para avaliar e, eventualmente, reformular o rumo do mesmo. Após a realização do treinamento teve início um processo mais cuidadoso de avaliação das rotinas abordadas.

Para avaliar a rotina Anotação de Enfermagem utilizamos uma análise de prontuários. Escolhemos, aleatoriamente, 10 prontuários anteriores e 10 posteriores ao treinamento sobre este tema e fizemos uma comparação entre ambos, levando em conta o número de palavras e o conteúdo da Anotação de Enfermagem de um plantão de 24 horas. Os resultados estão apresentados na Tabela 3.

Analisando a Tabela 3, podemos observar que:

- o número de palavras usadas na Anotação de Enfermagem apresentou uma elevação significativa;

- as partes, ou conteúdos da anotação, foram mais abrangentes. A evolução do estado de saúde da criança que antes apareceu uma vez, depois, esteve incorporado em três dos prontuários avaliados. Higiene, cuidados gerais e descrição de procedimentos, anteriormente não haviam sido explicitados;

- os horários das anotações passaram a ser especificados com maior freqüência após o treinamento.

Quanto aos demais procedimentos, o tipo de avaliação utilizada foi a observação imediata das funcionárias durante a realização dos procedimentos treinados.

Com relação à Admissão e Identificação da Criança, as funcionárias procederam da maneira como faziam anteriormente, excetuando-se a identificação, que antes não era realizada no momento da admissão e, ap6s o treinamento, passou a ser realizada. Ainda quanto à admissão da criança, observamos que a anotação de enfermagem passou a conter dados referentes às condições gerais da criança, local de procedência e quem a acompanhava. Anteriormente, as únicas anotações se referiam apenas ao motivo da internação, peso e temperatura.

Quanto aos Sinais Vitais não observamos mudanças no comportamento das funcionárias; o único sinal vital anotado continuou a ser a temperatura, a não ser em caso de prescrição médica. 
Tabela 3 - Distribuição do número de palavras e partes da Anotação de Enfermagem, segundo prontuário, antes e após o treinamento.

\begin{tabular}{|c|c|c|c|c|c|}
\hline \multicolumn{3}{|c|}{ ANTES DO TREINAMENTO } & \multicolumn{3}{|c|}{ APÓS O TREINAMENTO } \\
\hline $\begin{array}{l}\mathrm{N}^{\circ} \text { DE } \\
\text { PALAVRAS }\end{array}$ & $\begin{array}{l}\text { PARTES DA } \\
\text { ANOTAÇAOO DE } \\
\text { ENFERMAGEM }\end{array}$ & $\begin{array}{l}N^{\circ} \text { DO } \\
\text { PRONTUÁRIO }\end{array}$ & $\begin{array}{l}\text { N DE } \\
\text { PAIAVRAS }\end{array}$ & $\begin{array}{l}\text { PARTES DA } \\
\text { ANOTACAO DE } \\
\text { ENFERMAGEM }\end{array}$ & $\begin{array}{l}\text { N DO } \\
\text { PRONTUÁRIO }\end{array}$ \\
\hline 13 & $\begin{array}{l}\text { - ASSINATURA } \\
\text { - ELIMINAÇOES } \\
\text { - TEMPERATURA }\end{array}$ & $426694-1$ & 20 & $\begin{array}{l}\text { - HORÁRIO } \\
\text { - ALIMENTAÇAO } \\
\text { - EVOLUÇÃO } \\
\text { - DATA } \\
\text { - ASSINATURA }\end{array}$ & $243083-1$ \\
\hline 14 & $\begin{array}{l}\text { - ESTADO GERAL } \\
\text { - ELIMINAÇOES }\end{array}$ & $242680-1$ & 28 & $\begin{array}{l}\text { - TEMPERATURA } \\
\text { - ELIMINAÇES } \\
\text { - ALIMENTAÇAO } \\
\text { - ASSINATURA } \\
\text { - HORARIO }\end{array}$ & $235800-2$ \\
\hline 10 & $\begin{array}{l}\text { - HORÁRIO } \\
\text { - ESTADO GERAL } \\
\text { - ASSINATURA }\end{array}$ & $242674-1$ & 30 & $\begin{array}{l}\text { - DATA } \\
\text { - HIGIENE } \\
\text { - TEMPERATURA } \\
\text { - ALIMENTAÇAO } \\
\text { - ELIMINACOES } \\
\text { - ASSINATURA }\end{array}$ & 236758.4 \\
\hline 8 & $\begin{array}{l}\text { - PEDIDO DE } \\
\text { EXAME } \\
\text { - ASSINATURA }\end{array}$ & $242656 \cdot 1$ & 16 & $\begin{array}{l}\text { - AVALIACAOO } \\
\text { - ESTADO GERAL, } \\
\text { - SINAIS VITAIS } \\
\text { - ASSINATURA }\end{array}$ & $266266-6$ \\
\hline 12 & $\begin{array}{l}\text { - TEMPERATURA } \\
\text { - ALIMENTAÇAO } \\
\text { - ELIMINAÇAO } \\
\text { - ASSINATURA }\end{array}$ & $242655 \cdot 1$ & 25 & $\begin{array}{l}\text { - DATA } \\
\text { - HORARIO } \\
\text { - TEMPERATURA } \\
\text { - ESTADO GERAL } \\
\text { - EVOLUÇÃO } \\
\text { - ASSINATURA }\end{array}$ & $243193-1$ \\
\hline 12 & $\begin{array}{l}\text { - TEMPERATURA } \\
\text { - ALIMENTAÇAO } \\
\text { - ELIMINAÇAO }\end{array}$ & $242656 \cdot 1$ & 20 & $\begin{array}{l}\text { - HORÁRIO } \\
\text { - TEMPERATURA } \\
\text { - ALIMENTAÇAO } \\
\text { - ELIMINAÇESS } \\
\text { - ASSINATURA }\end{array}$ & 237643.8 \\
\hline 13 & $\begin{array}{l}\text { - ESTADO GERAL } \\
\text { - ALIMENTAÇAO } \\
\text { - TEMPERATURA } \\
\text { - ELIMINAÇÓES }\end{array}$ & $242651-1$ & 25 & $\begin{array}{l}\text { - CUIDADOS GERAIS } \\
\text { - TEMPERATURA } \\
\text { - ALIMENTAÇÃO } \\
\text { - ELIMINAÇÃO } \\
\text { - EVOLUÇÃO } \\
\text { - ASSINATURA }\end{array}$ & $232455-4$ \\
\hline 10 & $\begin{array}{l}\text { - ALIMENTAÇÃO } \\
\text { - EVOLUÇAO } \\
\text { - TEMPERATURA } \\
\text { - ELIMINAÇAO } \\
\text { - ASSINATURA }\end{array}$ & $242639-1$ & 15 & $\begin{array}{l}\text { CUIDADOS GERAIS } \\
\text { TEMPERATURA } \\
\text { - ALIMENTAÇAO }\end{array}$ & 236612.4 \\
\hline 6 & $\begin{array}{l}\text { ANOTAÇAO DE } \\
\text { ALTA }\end{array}$ & $242631-1$ & 16 & $\begin{array}{l}\text { - ESTADO GERAL } \\
\text { - TEMPERATURA } \\
\text { - ELIMINAÇÁO } \\
\text { - HORÁRIO } \\
\text { - ASSINATURA }\end{array}$ & $243183-1$ \\
\hline 9 & $\begin{array}{l}\text { - ALIMENTAÇ̃̃O } \\
\text { - ELIMINAÇOES } \\
\text { - ASSINATURA }\end{array}$ & $242675-1$ & 11 & $\begin{array}{l}\text { - TEMPERATURA } \\
\text { - PROCEDIMENTOS } \\
\text { - DATA } \\
\text { - ASSINATURA }\end{array}$ & $242721-2$ \\
\hline
\end{tabular}


Durante a Passagem de Plantão, tivemos a oportunidade de observar três funcionárias. Duas delas realizaram o procedimento conforme havia sido discutido durante o treinamento, exceto quanto à utilização do prontuário: foi realizado na enfermaria, junto ao leito da criança, com a folha de controles nas mãos. Durante a mesma, relatou-se a evolução da criança e os cuidados gerais prestados. A terceira funcionária realizou a passagem de plantão na enfermaria, junto ao leito, sem a folha de controles, sem o prontuário e com seguidas interrupções.

O procedimento de Sondagem Nasogástrica não foi realizado na unidade durante o período de avaliação, não podendo assim ser avaliado. Contudo, uma mudança foi observada quanto à rotina de coleta de lavado gástrico: esta passou a ser feita às 6:00 horas, de acordo com o que foi discutido. Isto foi constatado, observando a existência de uma folha afixada em um dos berços, na qual havia especificações a respeito do horário da coleta. Antes do treinamento, colhia-se o lavado gástrico em qualquer horário.

Quanto ao tópico Precauções Universais, que a princípio não fazia parte dos temas a serem abordados, e que portanto, não consta do Manual, consideramos importante abordá-lo, devido ao fato da necessidade ter emergido durante as atividades e, também porque as funcionárias o desconheciam. Para sua avaliação, detivemos-nos a observar alguns procedimentos. Notamos que, na realização de técnicas como aspiração e cuidados gerais (higiene da criança, por exemplo), as funcionárias passaram a utilizar luvas com maior freqüência, porém, para a punção venosa continuaram não usando-as, referindo ser desconfortável. Quanto às demais medidas de proteção, não observamos sua adoção.

Além do que já foi citado, consideramos importante conhecer a percepção que a equipe de enfermagem teve sobre o treinamento. Achamos que a melhor forma de fazê-lo seria através de entrevistas. Entrevistamos funcionárias dos quatro plantões e a enfermeira, totalizando 12 entrevistas.

Das entrevistas, pudemos apreender que a maioria das funcionárias estão abertas a este tipo de atividade e consideraram importante aprender e relembrar: "Ah, eu gostei bastante do pessoal, achei bacana porque foi um momento que nós pudemos sentar, discutir, relembrar alguma coisa e aprender também..." (Plantão Noturno Impar - 2Funcionária.)

Percebemos também que a maioria das entrevistadas referem-se à necessidade de outros treinamentos, sugerindo outros temas. "Seria bom também se tivesse mais cursinhos como este. Que nem o tema curativo..." (Plantão Noturno Par - 2 Funcionária.); “... eu sinto dúvida em colocar um abocath em criança e fazer uma sondagem vesica l...” (Plantão Noturno Par - 3 Funcionária.); “... por exemplo... o expurgo para mim seria bastante interessante... a gente não tem muito contato lá, com a solução que está sendo usada, tudo, eu tô assim praticamente fora dessas coisas..." (Plantão Norturno Ímpar - 2 Funcionária.) 
As funcionárias entrevistadas falaram sobre a importância da conscientização da assistência adequada à criança. "As vezes a pessoa entra e não têm toda aquela experiência, às vezes uns têm o curso e outros não têm, às vezes a pessoa aprende pelos outros, assim vindo de uma pessoa mais esclarecida, mais atualizada, ela fica consciente dos deveres da gente com o cuidar do paciente $e$ as vezes alguma coisa que está meio esquecida, assim a gente pode por em prática depois disso" (Plantão Noturno Impar - 3Funcionária.); “... Porque muitas vezes a criança chega no setor e se a gente não sabe nada, quais os cuidados a tomar, pode até colocar em risco a vida do paciente. Então sendo assim, a gente orientada, a gente tá pronta para dar um bom atendimento."(Plantão Diurno Par - 2 Funcionária).

Em relação à percepção do resultado do treinamento, tanto uma das auxiliares de enfermagem, quanto a enfermeira da unidade relataram uma mudança após o treinamento. "... estava ficando assim muita coisa sem ser feita, como a anotação de enfermagem que estava deixando de fazer, voltaram a fazer anotação direitinho; estavam deixando de colocar a pulseirinha nas crianças ...". (Plantão Noturno Ímpar - 2 Funcionária.); "Uma coisa muito marcante para elas, por exemplo, foi a anotação de enfermagem. Se você pega pranchetas, papeletas de cada uma delas, você vai ver que elas estão anotando...". (Plantão Diurno Diário - Enfermeira).

A avaliação das funcionárias em relação à didática utilizada no treinamento mostrou a importância da metodologia para se atingir os objetivos propostos. "... achei ótimo, foi didático acho que para elas que nunca, algumas nem terminaram o colégio, tinham medo das perguntas que não tinham prática ... A terminologia não foi complicada, foi bem clara, do dia-a-dia que elas usam aqui..." (Plantão Diurno Diário - Enfermeira).

Todas as pessoas entrevistadas apontaram claramente a necessidade de treinamento contínuo dentro da unidade; o que foi ao encontro da nossa proposta de treinamento em serviço e de início de sistematização da assistência de enfermagem.

\section{7 - CONSIDERAÇÕES FINAIS}

Entendemos que os resultados obtidos, através da realização deste trabalho, referem-se ao campo utilizado para estudo, à realidade vivenciada por aquelas funcionárias, àquela instituição. Tanto a elaboração do Manual de Rotinas como o Treinamento basearam-se nesse mesmo contexto.

Temos claro que a avaliação realizada através da análise dos prontuários oferece um esboço geral e restrito dos resultados obtidos através do treinamento.

Consideramos que a produção de resultados efetivos depende igualmente de treinamento, supervisão e avaliação contínua, porém, com o nosso trabalho, 
tivemos a oportunidade de mostrar que existe a possibilidade de mudanças de comportamentos e hábitos das funcionárias, quando as mesmas participam ativamente de um processo de educação continuada.

Percebemos as entrevistas como uma parte bastante importante deste trabalho, pois não há treinamento que funcione quando a funcionária não está envolvida e, através delas, detectamos este interesse.

O treinamento de todas as rotinas que constam do Manual não fez parte de nossos objetivos, porém, durante todo o processo deixamos claro que o Manual de Rotinas estaria à disposição na Unidade para consulta, sempre que as mesmas sentissem necessidade.

Os resultados do trabalho vão ao encontro das colocações dos autores citados na introdução, tornando claro que a capacitação dos profissionais favorece o desenvolvimento e promove as condições para que eles realizem uma assistência de enfermagem de qualidade, dentro das possibilidades encontradas.

Acreditamos que, para garantir qualidade, o processo de sistematização deva ser buscado em toda sua complexidade. Esperamos que, com a realização deste trabalho, tenhamos iniciado um processo de Sistematização da Assitência de Enfermagem e um trabalho de Educação Continuada dentro da Unidade Pediátrica em questão.

\section{REFERÊNCIAS BIBLIOGRÁFICAS}

AUGUSTO, M.; NODA, M. Enfermeira pediátrica em terapia intensiva. São Paulo, Sarvier, 1987.

CAMPESTRINI, S. Súmula pediátrica. Curitiba, EDUCA, 1991.

CURSINO, M.R. et al. Assistência de enfermagem em pediatria. São Paulo, Sarvier, 1982.

KURCGANT, P. (Coord.) et al. Administracão em enfermagem. São Paulo, EPU, 1991.

SCHIMITZ, E.M. et al. A enfermagem em pediatria e puericultura. São Paulo, Atheneu, 1989.

\section{BIBLIOGRAFIA}

CAMPEDELLI, M.C. et al. Escara: problema na hospitalização. São Paulo, Ática, 1987.

CASTELHANOS, B.P. Iniecđes: modos e métodos. São Paulo, Ática, 1987.

DUTRA, V.O, et al Enfermagem em cardiologia , Săo Paulo, Sarvier/EDUSP, 1981.

HENDRIKS, H.M. Manual de organizacão e avaliacão do servico de enfermagem. 4. ed. São

Paulo, USC, s.d. 
HORNEMANN, G.V. Procedimentos básicos de enfermagem. São Paulo, EDUSP, 1977.

HUGNES, W.T.; BUESCHER, E.S. Procedimentos técnicos em pediatria. 2. ed. Rio de Janeiro, Interamericana, 1983.

KAWAMOTO, E.; FORLER, J.I. Fundamentos de enfermagem. Sao Paulo, EDUSP, 1986.

KOCH, R.M.; MOTTA, H.S.; WACTER, R.L. Técnicas de enfermagem. Curitiba, Litero Técnicas, 1978.

MAMEDE, M.V.; CARVALHO, E.C.; CUNHA, A.M.P. Técnicas em enfermagem. São Paulo, Sarvier, 1984.

MARCONDES, E.; ALCÂNTARA, A.P. Pediatria básica. Sao Paulo, Sarvier, 1980.

MARLOW, P.R.; REDDINE, B.A. Enfermaria pediátrica. Buenos Aires, 1991.

PASSOS, A.R.C. Aplicações via parenteral. Feira de Santana, Grafinort, 1990.

SCHATSMAN, S. Medicamentos em pediatria. Sao Paulo, Sarvier, 1988.

SOUZA, E.F. Administracão de medicamentos e preparo de solucões. Rio de Janeiro, Cultura Médica, 1976.

SOUZA, E.F. Novo manual de enfermagem. Rio de Janeiro, Cultura Médica, 1976.

STENSSCHNEIDER, J. et al. Cadernos de enfermagem pediátrica. Rio de Janeiro, Mason, 1981.

VEIGA, P.A.; CROSSETTI, M.O. Manual de técnicas de enfermagem. Porto Alegre, Cuzzato Editores, 1986.

WHALEY, L.F.; WONG,D.L. Enfermagem pediátrica - elementos essenciais à intervençăo efetiva . 2. ed. Rio de Janeiro, Guanabara Koogan, 1989.

SANTOS, E.R. Nursing assistence in pediatric unit: an initial systematization proposal. Rev.Esc.Enf. USP, v.31, n.1 p. 36-50, apr. 1997.

The present work reports an initial process of nursing assistance systematization developed in a Pediatric Unit of a medium size hospital in São Carlos - SP, considering the actual human resources and the type of approach adopted by the institution on the assistance given to the hospitalized child. A Routine Manual was elaborated based on the literature and with the staff participation, and after that, a training was realized with these employees, starting from routines previously sketched, using groupal dynamics that looked forward to rescue the knowledge that they already had about the themes. The work considered the employees opinion about their participation in the systematization process.

UNITERMS: Pediatric Assistance Systematization. Participative Groupal Dynamic. Training in Service. 